\title{
When imaging meets neurophysiology: the value of navigated transcranial magnetic stimulation for preoperative neurophysiological mapping prior to brain tumor surgery
}

\author{
Giovanni Raffa, MD, PhD, ${ }^{1}$ Maria Catena Quattropani, PsyD, ${ }^{2}$ and Antonino Germanò, MD'1 \\ ${ }^{1}$ Division of Neurosurgery, BIOMORF Department, University of Messina, Italy; and ${ }^{2}$ Department of Clinical and Experimental \\ Medicine, University of Messina, Italy
}

\begin{abstract}
Maximal safe resection is the modern goal for surgery of intrinsic brain tumors located in or close to brain eloquent areas. Nowadays different neuroimaging techniques provide important anatomical and functional information regarding the brain functional organization that can be used to plan a customized surgical strategy to preserve functional networks, and to increase the extent of tumor resection. Among these techniques, navigated transcranial magnetic stimulation (nTMS) has recently gained great favor among the neurosurgical community for preoperative mapping and planning prior to brain tumor surgery. It represents an advanced neuroimaging technique based on the neurophysiological mapping of the functional cortical brain organization. Moreover, it can be combined with other neuroimaging techniques such as diffusion tensor imaging tractography, thus providing a reliable reconstruction of brain eloquent networks. Consequently, nTMS mapping may provide reliable noninvasive brain functional mapping, anticipating information that otherwise may be available to neurosurgeons only in the operating theater by using direct electrical stimulation. The authors describe the reliability and usefulness of the preoperative nTMS-based approach in neurosurgical practice, and briefly discuss their experience using nTMS as well as currently available evidence in the literature supporting its clinical use. In particular, special attention is reserved for the discussion of the role of nTMS as a novel tool for the preoperative neurophysiological mapping of motor and language networks prior to surgery of intrinsic brain tumors located in or close to eloquent networks, as well as for future and promising applications of nTMS in neurosurgical practice.
\end{abstract}

https://thejns.org/doi/abs/10.3171/2019.9.FOCUS19640

KEYWORDS brain tumors; DTI tractography; diffusion tensor imaging; eloquent areas; navigated transcranial magnetic stimulation; neurophysiological mapping; presurgical planning

$\mathrm{N}$ EUROIMAGING techniques have shown great advancements in the past few decades, leading to the development of very complex and fascinating morphological in vivo studies of brain structure. These advancements have been progressively translated in clinical practice and have resulted in outstanding support of the neurosurgical decision-making process, especially prior to brain tumor surgery ${ }^{76}$ Nowadays, high-field MRI as well as other MR techniques ranging from diffusion tensor imaging (DTI) to MR spectroscopy (MRS) provide detailed information regarding brain structural organization at a cortical, subcortical, and molecular level. In many cases these data are essential prior to brain tumor surgery, especially for defining a customized presurgical plan based on the spatial relationship between the tumor and the surrounding important anatomical landmarks.

Nevertheless, the recent hodotopic revolution disclosed the complexity of the brain's functional organization. ${ }^{6}$ Anatomical landmarks are important of course, but they do not always correspond to the hypothesized neurological functions, especially because of the possibility of tumorinduced neuroplasticity. ${ }^{8}$ In these settings, neuroimaging

ABBREVIATIONS AF = arcuate fascicle; $C S T=$ corticospinal tract; $D C S=$ direct cortical stimulation; $D E S=$ direct electrical stimulation; $D S S=$ direct subcortical stimulation; $\mathrm{DTI}=$ diffusion tensor imaging; FA = fractional anisotropy; $\mathrm{fMRI}=$ functional MRI; IFOF = inferior frontooccipital fascicle; ILF = inferior longitudinal fascicle; MEP = motor evoked potential; M1 = primary motor cortex; $\mathrm{nTMS}$ = navigated transcranial magnetic stimulation; RMT = resting motor threshold; ROI = region of interest; $\mathrm{rTMS}=$ repetitive transcranial magnetic stimulation; SLF = superior longitudinal fascicle; UF = uncinate fascicle.

SUBMITTED August 1, 2019. ACCEPTED September 4, 2019.

INCLUDE WHEN CITING DOI: 10.3171/2019.9.FOCUS19640. 
moved toward the development of advanced techniques for the identification of the "functional" brain architecture. ${ }^{34}$ Many indirect approaches to map brain functional organization have been implemented, such as functional MRI (fMRI) or magnetoelectroencephalography (MEG). Nevertheless, the ability of these techniques to detect neural activity with a high spatial and temporal resolution has been questioned..$^{9,35,66}$

As a matter of fact, most knowledge about brain functional architecture derives from direct electrical stimulation (DES), at both a cortical (direct cortical stimulation, DCS) and subcortical (direct subcortical stimulation, DSS) level during awake surgery. ${ }^{10}$ From Penfield to the present time, brain functional organization has been primarily studied using the same "language" as that of the nervous system (that is, electrophysiology). ${ }^{10}$ Indeed, intraoperative neurophysiological mapping by DES is still considered to be the gold standard to identify functional brain structures and is essential for achieving the maximal safe resection of supratentorial intrinsic tumors, ${ }^{65}$ as well as many posterior fossa ${ }^{75}$ and spinal neoplasms. ${ }^{67,68}$

In the last decade a new mixed technique merging advanced imaging and electrophysiology, namely navigated transcranial magnetic stimulation (nTMS), has progressively gained favor among the neurosurgical community for studying and understanding brain functional organization. ${ }^{45,53}$ nTMS provides a reliable combination of advanced imaging with the direct electrophysiological evaluation of neural activity, thereby representing a new approach for a noninvasive preoperative neurophysiological mapping of brain functional organization. It may be considered a valuable functional adjunct to preoperative planning before brain tumor surgery, especially in the case of intrinsic lesions located in eloquent areas.

The aim of this paper was to describe the usefulness of nTMS in neurosurgical practice; to discuss its value in answering everyday complex clinical questions prior to brain tumor surgery, even in combination with other neuroimaging techniques; and to summarize evidence in the current literature supporting its use in neurosurgical departments.

\section{Technical Details for nTMS}

Transcranial magnetic stimulation is a well-established electrophysiological technique consisting of noninvasive brain stimulation performed using a magnetic field that is able to induce a secondary ionic current in the cortical neurons ${ }^{64}$ From a technical point of view, a primary electrical current is delivered to a magnetic coil, resulting in the induction of a magnetic field. The coil (usually a figure-of-eight coil) is applied over the scalp of patients. The induced magnetic field reaches the brain cortex, thus inducing a modification of the neuronal excitability and resulting in an excitatory or inhibitory effect depending on the specific stimulation parameters used. The modifications of cortical activity and excitability can be measured at a cortical level and/or recorded at the peripheral muscles. $^{63}$

The novel aspect of nTMS is the combination with neuronavigation. The TMS machine is connected to a dedicated station equipped with an optical navigation system (Fig. 1). This combination enables the navigation of the stimulation coils over an accurate 3D rendering of the patient's brain MRI scan (Fig. 2). After the coregistration process, the nTMS coil movements are visualized in real time over the 3D rendering of the patient's brain MRI scan, thereby enabling the operator to map specific cortical gyri with a high spatial accuracy. The adjunct of navigation converts the procedure into an advanced neuroimaging technique, and represents the main advantage over the traditional TMS mapping which relies only on external scalp landmarks. nTMS can be performed using an excitatory or inhibitory paradigm according to the specific brain functional areas that need to be investigated.

\section{nTMS-Based Motor Mapping}

The nTMS excitatory paradigm is usually used for mapping the motor network-it is based on a single-pulse stimulation applied over the primary motor cortex (M1). It results in motor evoked potentials (MEPs) that can be recorded at the contralateral peripheral muscles by using surface electromyography electrodes. The MEPs' amplitude and latency can be measured to investigate the integrity of the motor descending network. Usually a single muscle for each contralateral body segment (face, upper limb, lower limb) is chosen. ${ }^{17}$ Stimulation is performed after the measurement of the resting motor threshold (RMT), which is defined as the lowest stimulation intensity capable of eliciting a response in a given relaxed muscle in 5 of 10 stimulations.$^{63}$ Then, the stimulation is applied using an intensity of $110 \%$ of the RMT. Each single stimulation is visualized as an nTMS spot over the 3D rendering of the patient's brain MRI scan, and the characteristics of each single MEP are recorded. This enables the offline analysis of the motor responses according to their amplitude and latency. The final result is the nTMS map of the M1, with its somatotopic motor organization (Fig. 3).

\section{nTMS Combination With DTI Fiber Tracking}

The nTMS map of M1 can be used as a seeding region for the DTI fiber tracking of the corticospinal tract (CST). The standard anatomically based DTI fiber tracking relies on the selection of specific anatomical structures as regions of interest (ROIs) for seeding the tracking of the pyramidal tract. ${ }^{1}$ That may increase the interoperator variability of results, because different seeding ROIs may be selected by different operators. ${ }^{7}$ Moreover, one of the most important drawbacks of the anatomically based approach, especially when using deterministic algorithms-which are usually available in the most common neuronavigation systems - is the difficult tracking of the most lateral portion of the CST, which contains the corticobulbar fibers originating from the cortical motor representation of face muscles. ${ }^{49}$

The nTMS-based DTI fiber tracking of the CST has the advantage of using functionally verified cortical landmarks as seeding ROIs. Indeed, each single spot of the nTMS map corresponds to a functional region of M1 that has been stimulated, resulting in an MEP. That reduces the interoperator variability of results. Moreover, the nTMSbased approach enables the visualization of the corti- 




FIG. 1. Picture of the nTMS system used for preoperative functional mapping prior to brain tumor surgery.

cobulbar component of the CST, thereby making visible the most lateral part of the pyramidal tract, even using a deterministic approach. ${ }^{59}$

\section{Neurosurgical Use-Technical Details and Rationale}

Neurosurgeons with some electrophysiological expertise can easily perform the motor mapping procedure: it usually lasts approximately 15-30 minutes. MEP characteristics must be analyzed, removing motor responses with latency shorter than $10 \mathrm{~mA}$ and/or longer than 35 $\mathrm{mA}$ from the final map, as well as MEPs with low amplitude $(<100 \mu \mathrm{V})$ to avoid false-positive responses. ${ }^{17}$ Those false-positive responses can be caused by the patient's involuntary movements or by electrophysiological artifacts. In patients with severe motor deficits, nTMS may fail to elicit MEPs due to the impairment of the motor pathway. In those cases, a possible solution is to try to record MEPs from different muscles than those usually mapped, such as biceps or deltoid for the upper limb, quadriceps for the
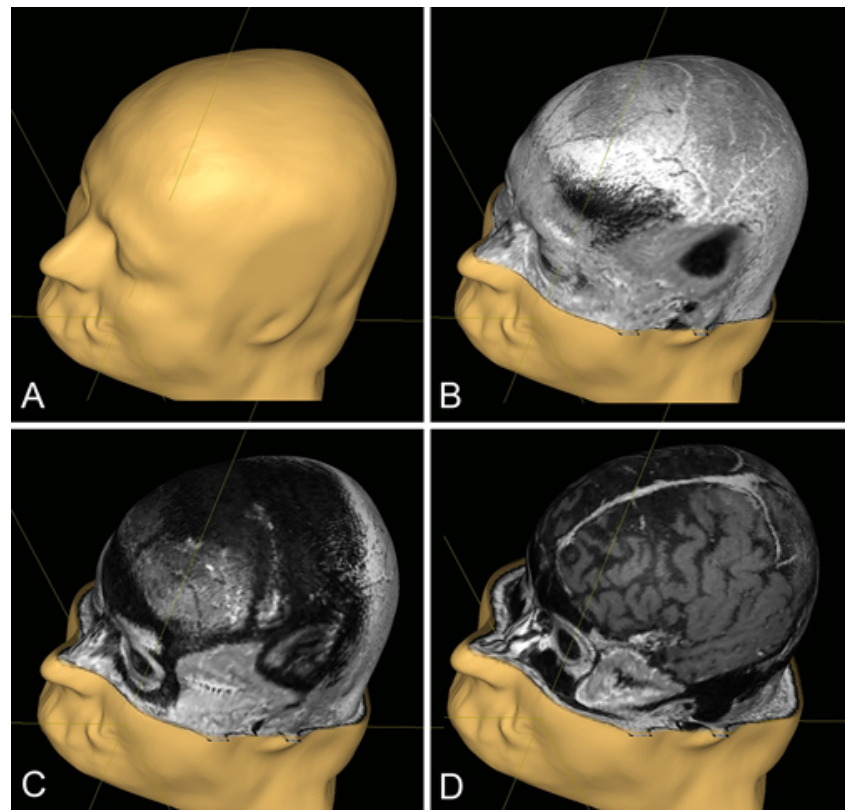

FIG. 2. Example of the 3D rendering of the patient's head MRI scan. A-D: The progressive increase of the depth of rendering allows for the final visualization of an accurate reconstruction of brain cortical surface.

lower limb, and the nasal or buccinator muscles for the face. ${ }^{17}$ In some cases, this strategy may lead to a successful mapping even in hemiplegic patients. ${ }^{47}$

Once the nTMS map of M1 is obtained, it can be exported in DICOM format to the neuronavigation system equipped with tractography software. Here, it can be used to perform the somatotopic DTI fiber tracking of the CST. Each single area containing the nTMS-verified motor cortical representation of the face, upper-limb, and lowerlimb muscles can be separately selected as first seeding ROI and combined with a second ROI positioned in the cerebral peduncle or the internal capsule. ${ }^{4}$ The most commonly used parameters are as follows: fractional anisotropy (FA) threshold $=0.20$, which can be progressively lowered in case of excessive perilesional edema (no less than $75 \%$ of the FA threshold): ${ }^{14,50}$ and maximum directional change $=45^{\circ}$.

The nTMS-based approach results in an advanced functionally based reconstruction and visualization of the motor pathway (Video 1).

VIDEO 1. Example of the preoperative nTMS-based reconstruction of the motor network in 2 cases of right frontal glioblastomas. In the first case, the motor network is simply dislocated posteriorly by the tumor, thus reducing risks of resection. In the second case, the CST is clearly infiltrated by the lesion, making surgery at high risk for the occurrence of postoperative motor deficits. Copyright Giovanni Raffa. Published with permission. Click here to view.

This reconstruction enables neurosurgeons to analyze the spatial relationship between the tumor and the motor network (M1 + CST), thus increasing the awareness about surgical risks. nTMS-based information therefore enables customized, neurophysiologically based presurgical planning that may really help in defining the best surgical strategy for maximal safe resection of motor-eloquent intrinsic tumors (Fig. 4). 


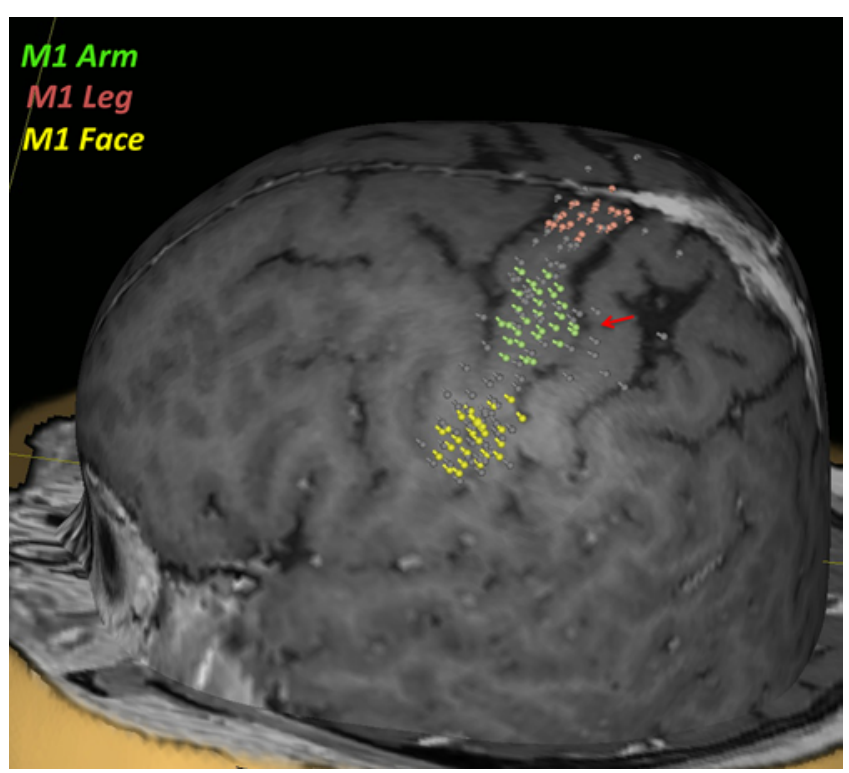

FIG. 3. The final result of the nTMS mapping. The somatotopic organization of the M1 is identified by colors: the area corresponding to the motor cortical representation of leg muscles is indicated by red spots, the area of arm muscles by green spots, and the face muscles area by yellow spots. The red arrow indicates the omega sign corresponding to the rolandic fissure.

Moreover, the nTMS-based reconstruction of M1 and CST may also be used during surgery: the M1 map and the CST may be merged with the anatomical brain MRI scan and visualized on the neuronavigation screen, thus providing a real-time feedback about the exact location of the motor network during surgery. That may help surgeons in tailoring the craniotomy, eventually guiding DES and tumor resection ${ }^{51}$ (Fig. 5).

\section{Neurosurgical Use-Current Evidence}

The first paper reporting the introduction of nTMS motor mapping for preoperative planning in patients with brain tumor was published in 2009.45 Thereafter, other studies demonstrated that findings from nTMS motor mapping were similar to the ones achieved by DCS, reporting a distance ranging between 1.1 and $14.8 \mathrm{~mm}$ for the hotspots comparison. . $^{12,32,42,46}$ The highest distance was measured for the hotspots of lower-limb muscles. ${ }^{32}$

Some studies analyzed the reliability of the nTMS motor mapping in comparison with other neuroimaging techniques. Coburger et al. demonstrated that the mean accuracy to localize M1 was higher when using nTMS than fMRI in 30 consecutive patients with rolandic brain tumors. ${ }^{3}$ Forster et al. reported that distances between nTMS and DCS spots were significantly smaller than those between fMRI activation regions and DCS spots. ${ }^{11}$ Finally, Mangraviti et al. confirmed previous studies, documenting that the mean distance from DCS motor sites was shorter when using nTMS than when using fMRI. ${ }^{38}$ Collectively, these data highlight the higher spatial accuracy of nTMS motor mapping compared to the widely diffused fMRI.

Later studies analyzed how the routine use of nTMS motor mapping could impact surgical strategy for brain tumor resection. In a series of 73 patients with motor-eloquent tumors, Picht et al. reported that nTMS mapping had a helpful impact on surgical strategy in just more than half of patients. ${ }^{48}$ Rizzo et al., in a series of 17 patients with rolandic tumors, reported that nTMS mapping correctly identified M1 in $88.2 \%$ of cases, inducing a modification of the planned surgical strategy in $29.4 \%$ of cases ${ }^{60}{ }^{6 i}$ nally, Krieg et al. demonstrated that nTMS motor mapping led to a conversion of the treatment approach in $68.5 \%$ of cases in a series of 100 patients with motor-eloquent lesions. ${ }^{31}$

The possibility for the use of nTMS cortical information as seeding ROIs for DTI fiber tracking of the CST has been reported by several studies., ${ }^{4,145}$ The nTMS-based CST reconstruction seems more accurate than the standard DTI tractography, correlating well with DSS findings. ${ }^{4}$ Moreover, the intraoperative visualization of the nTMSbased reconstruction of the motor network may be useful to guide DES and tumor resection, allowing an easier identification of high-risk areas versus areas that can be safely resected (excluding limitations caused by brain shift). ${ }^{51}$ The eventual combination with intraoperative MRI may further improve the value of nTMS-based reconstruction of the motor pathway: it enables intraoperative DTI computation of the CST, thus reducing inaccuracy caused by brain shift, as well as enabling verification of the extent of tumor resection guided by nTMS findings and DES.

More recent studies clearly confirmed that nTMSbased planning may improve the treatment of brain tumors located in the central region. ${ }^{13,30,31,51,54,57} \mathrm{~A}$ recent meta-analysis concluded that nTMS motor mapping may lead to a reduced occurrence of postoperative permanent motor deficits, an increased extent of tumor resection, and a tailored surgical approach compared to standard surgery without nTMS mapping..$^{8}$ These results are probably due to the higher preoperative awareness of the functional anatomy and surgical risks, but they derive from observational studies only, and therefore must be interpreted with caution. Our single-center experience between 2012 and 2019 contributed to these literature findings; in a series of 228 patients with motor-eloquent tumors we achieved gross-total resection in $67.6 \%$ of cases, and permanent motor deficits ( 3 months postsurgery) occurred in $7.5 \%$ of patients (Table 1). Nevertheless, the first randomized clinical trial on the topic is still ongoing (https://clinicaltrials. gov/ct2/show/NCT02879682), and results have not been published yet.

\section{nTMS-Based Language Mapping}

As well as neurophysiological language mapping by DES during awake surgery, nTMS mapping of the language network relies on an inhibitory stimulation paradigm applied during the execution of a specific task designed for the exploration of different domains of the language function (articulation, naming, comprehension, etc.). A low-frequency repetitive stimulation is applied during the execution of a visual object naming test aiming to elicit language errors and/or disturbances according to a virtual transient lesion model. ${ }^{43}$ Briefly, the object naming task is performed using simple black-and-white pictures 
Raffa et al.
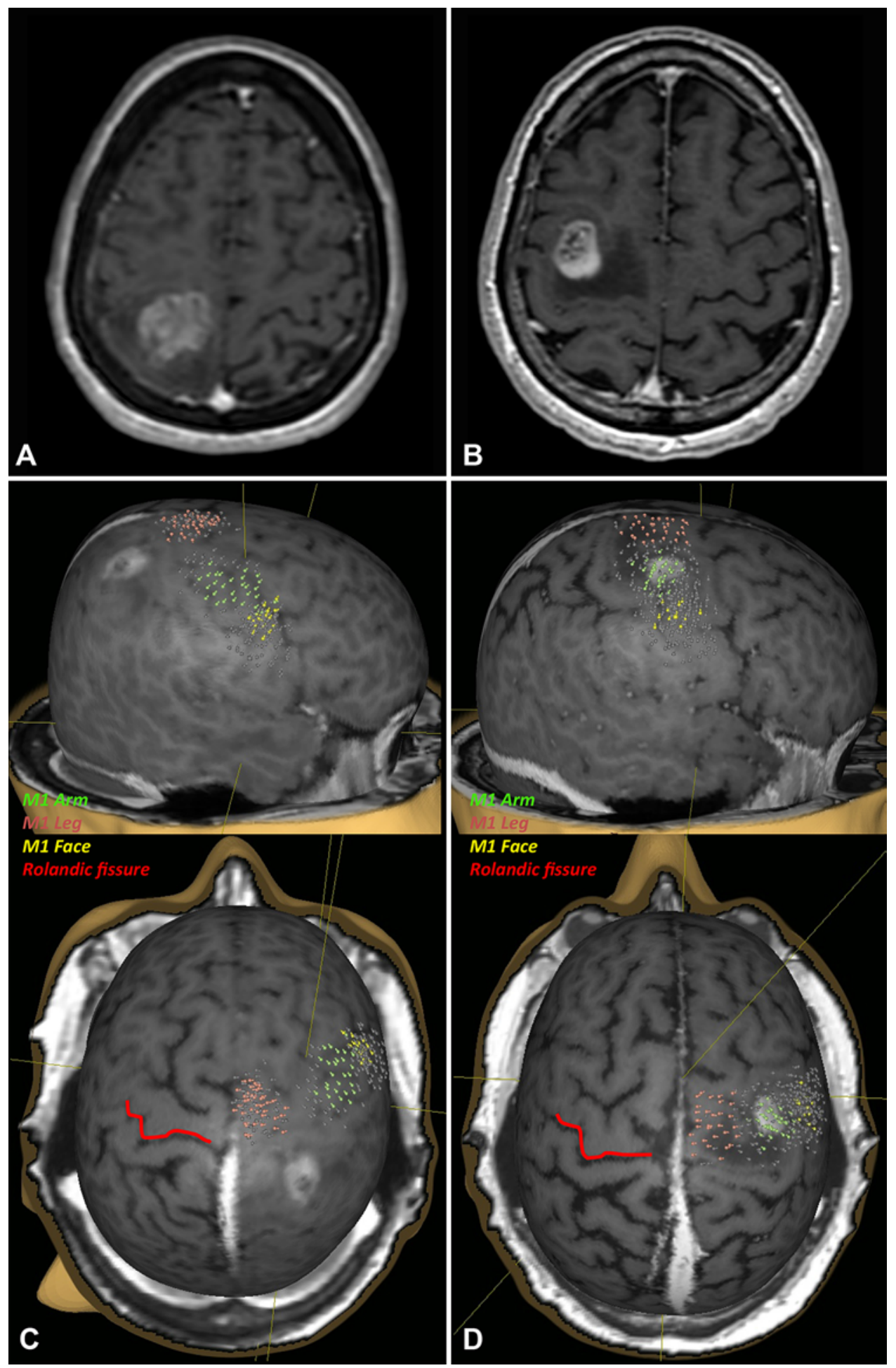

FIG. 4. Examples of 2 cases of suspected motor-eloquent intrinsic brain tumors. A: The tumor seems to be located just posteriorly to the M1 according to the identification of the contralateral omega sign representing the sylvian fissure. B: The tumor seems to be located anteriorly to the M1 according to the contralateral omega sign. C: nTMS mapping disclosed that the tumor was far away from the M1, making surgical resection easier and safer. D: In the second tumor case, the lesion is just below the M1, making surgery at high risk for the occurrence of postoperative motor deficits. 
Raffa et al.

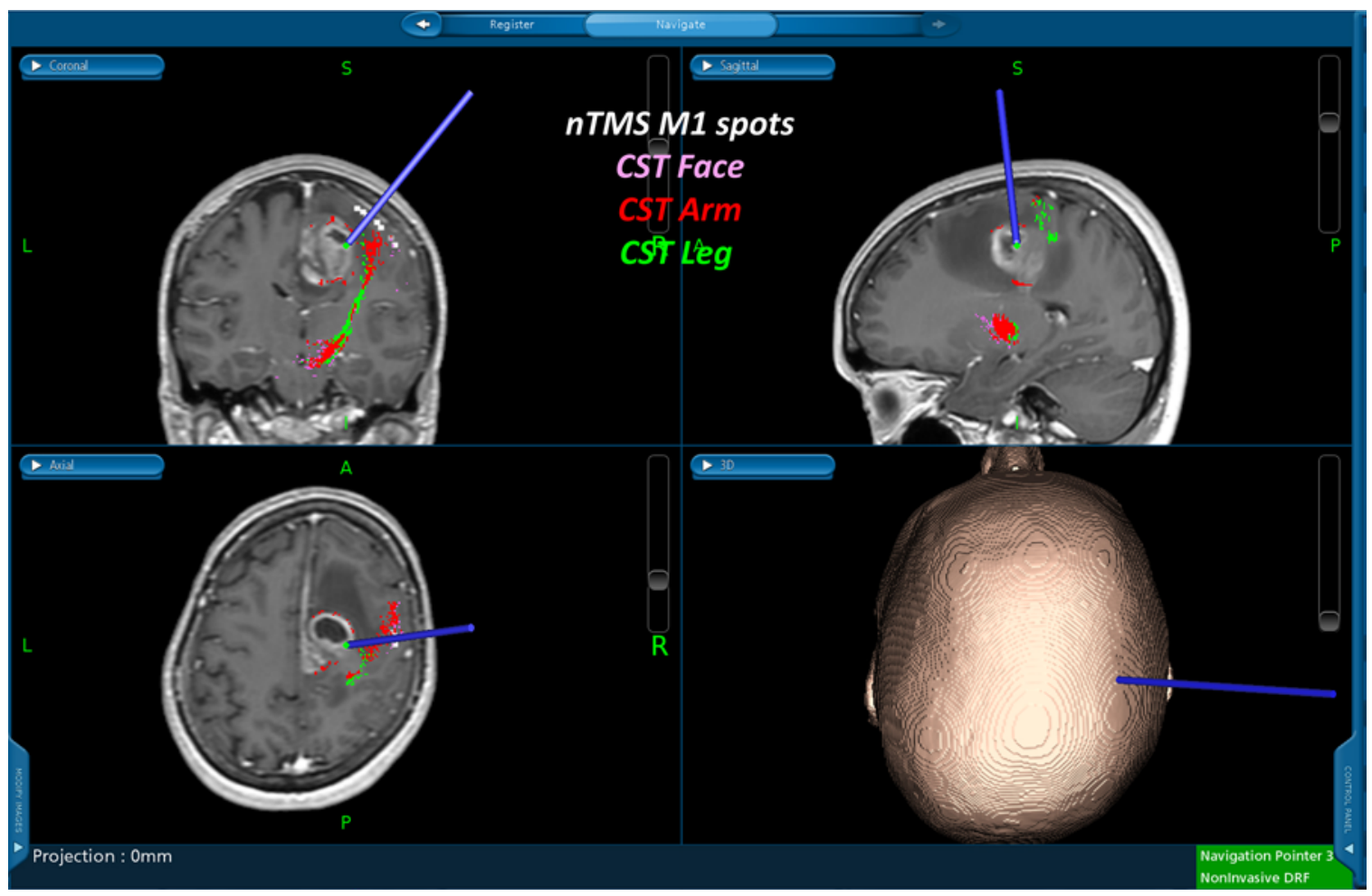

FIG. 5. Example of the intraoperative use of nTMS-derived reconstruction of the motor network as a guide to tailor DES and tumor resection thanks to its visualization on the navigation screen.

TABLE 1. Patient demographics and outcome after nTMS-based preoperative mapping was used at the University of Messina (2012-2019)

\begin{tabular}{lcc}
\hline & \multicolumn{3}{c}{ Value } \\
\cline { 2 - 3 } \multicolumn{1}{c}{ Characteristic } & $\begin{array}{c}\text { nTMS Motor Mapping-Tumors Close to } \\
\text { Motor Pathway }\end{array}$ & $\begin{array}{c}\text { nTMS Language Mapping-Tumors Close to } \\
\text { Language Pathways }\end{array}$ \\
\hline No. of patients & 228 & 107 \\
\hline Age in yrs, mean \pm SD & $53.9 \pm 13.9$ & $54.5 \pm 14.6$ \\
\hline Sex (\%) & $134(58.8)$ & $71(66.4)$ \\
\hline Male & $94(41.2)$ & $36(33.6)$ \\
\hline Female & & $62(57.9)$ \\
\hline Pathology (\%) & $166(72.8)$ & $15(14)$ \\
\hline High-grade gliomas & $40(17.5)$ & $14(13.1)$ \\
\hline Low-grade gliomas & $14(6.2)$ & $16(15)$ \\
\hline Metastases & $8(3.5)$ & $78(72.9)$ \\
\hline Other & & $24(22.4)$ \\
\hline Extent of resection (\%) & $154(67.6)$ & $4(3.7)$ \\
\hline Gross-total resection & $55(24.1)$ & $1(1)$ \\
\hline Subtotal resection & $14(6.1)$ & $6(5.6)$ \\
\hline Partial resection & $5(2.2)$ & \\
\hline Biopsy only & $17(7.5)$ & \\
\hline Permanent deficits at 3 mos postop (\%) & & \\
\hline Aldata & & \\
\hline
\end{tabular}

All data given as number of patients or tumors (\%) unless otherwise indicated. The "other" category of tumors includes mixed glioneuronal tumors, embryonal tumors, and hemangiomas. 


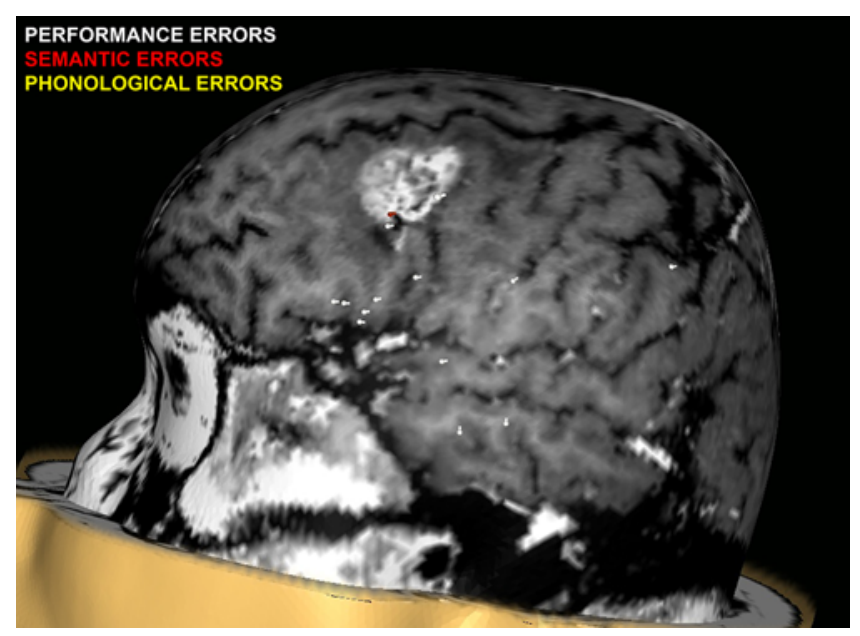

FIG. 6. Example of nTMS language mapping in the case of a left frontal glioblastoma. Some nTMS spots seem really close to the lesions (2 performance errors in white, and 1 semantic error in red). Therefore, resection could be at high risk for postoperative language deficits.

of common objects that are randomly shown on a screen in front of the patient. The patient is asked to correctly name these objects. A baseline test is performed 3 times without any stimulation to remove all pictures that are not properly named. Then, the task is repeated during the repetitive transcranial magnetic stimulation (rTMS). The rTMS trains are composed of 5 pulses at $5 \mathrm{~Hz}$ using an intensity of $100 \%$ of the ipsilateral RMT; if no naming errors occur, the number of pulses, their frequency, and their intensity are progressively increased.$^{29}$ Every single picture is displayed for $700 \mathrm{msec}$, with an interpicture interval of 2500 msec. rTMS starts simultaneously with the picture presentation to increase mapping sensitivity in the posterior perisylvian areas. ${ }^{29} \mathrm{~A}$ camera records the mapping procedure for the offline analysis of responses. Neuropsychologists compare patients' responses during rTMS stimulation versus baseline sessions. Naming errors that occur during the stimulation correspond to cortical language essential sites: they belong to the language networks and are classified in 3 categories consisting of performance, semantic, and phonological errors. ${ }^{5}$ Finally, each single stimulation spot is visualized over the $3 \mathrm{D}$ rendering of the patient's brain MRI scan, thus representing the final nTMS map of cortical language essential sites (Fig. 6).

\section{nTMS Combination With DTI Fiber Tracking}

The most important white matter tracts involved in the language networks consist of the superior longitudinal fascicle (SLF) and its major component, the arcuate fascicle (AF), belonging to the so-called dorsal stream and involved in the articulatory control of language. Other important tracts are the inferior frontooccipital fascicle (IFOF), the inferior longitudinal fascicle (ILF), and the uncinate fascicle (UF), belonging to the ventral stream and involved in semantic and phonological encoding. ${ }^{2}$

Several DTI tractography approaches have been reported for the computation of this fascicle network. The most commonly used technique is based on the choice of specific anatomical landmarks as seeding ROIs. ${ }^{1} \mathrm{Nev}-$ ertheless, it does not take into account the variability of functional landmarks that can be observed in patients with brain tumors; this variability is due to neuroplasticity phenomena and connectivity changes related to the presence of the tumor itself. ${ }^{6,8}$ Some authors have also pointed out the dangers of the use of DTI tractography in brain surgery, especially because the fibers' reconstruction is also dependent on the physician who decides where to put the ROIs for tracking.

To overcome this drawback, as well as for the motor pathway, the rTMS cortical map can be used as a functionally verified seeding ROI for the computation of the language network fascicles. Indeed, rTMS can transiently inhibit cortical essential sites, similarly to what is routinely performed during awake surgery by DES. That leads to the objective neurophysiological identification of language-eloquent cortical areas, thus reducing the interoperator variability of the DTI approach. Several approaches for nTMS-based language DTI fiber tracking have been described in detail, and were found to be feasible and accurate. ${ }^{41,50,52,56,72}$

\section{Neurosurgical Use-Technical Details and Rationale}

The mapping procedure usually takes between 20 and 40 minutes, but it needs the support of neuropsychologists. Indeed, the most difficult aspect of the procedure is the offline analysis of response errors induced by rTMS. The expertise of neuropsychologists is mandatory to disclose the occurrence of even minimal errors involving different language domains. Conversely, as well as during awake surgery, the lack of patients' verbal collaboration reduces the robustness of rTMS mapping. Nevertheless, in selected patients with a minimal verbal collaboration, rTMS mapping may be feasible even in cases of severe aphasia. ${ }^{73}$

Once the offline analysis has been performed, the rTMS language map can be easily exported in a DICOM format to a planning station or a navigation system. Tractography can be performed by selecting separately each single rTMS language spot or all spots together as a first seeding ROI, ${ }^{50}$ and a second anatomical ROI can be selected based on the specific fascicle to track. ${ }^{1}$ Tracking is usually performed using an FA threshold of 0.20 ; in case of excessive perilesional edema it can be lowered to achieve the best visualization of each fascicle (no less than $75 \%$ of the FA threshold); ${ }^{14}$ maximum directional change should be $45^{\circ}$ for IFOF and ILF, and up to $90^{\circ}$ for AF and UF. ${ }^{50}$

The rationale for the use of nTMS-based reconstruction of the language network consists in the possibility of performing customized presurgical planning in patients affected by intrinsic brain tumors located in the perisylvian region of the hemisphere specializing in language processing (Fig. 7). Moreover, as was previously described for the motor pathway, nTMS-based reconstruction may be displayed on the navigation screen and may serve as visual feedback during surgery, thus helping surgeons to preserve functional structures, ${ }^{56}$ especially if combined with intraoperative MRI. Finally, rTMS-based language mapping is especially useful in all those cases in which awake surgery cannot be performed, thereby representing the only functional information available for surgeons..$^{20,56}$ 


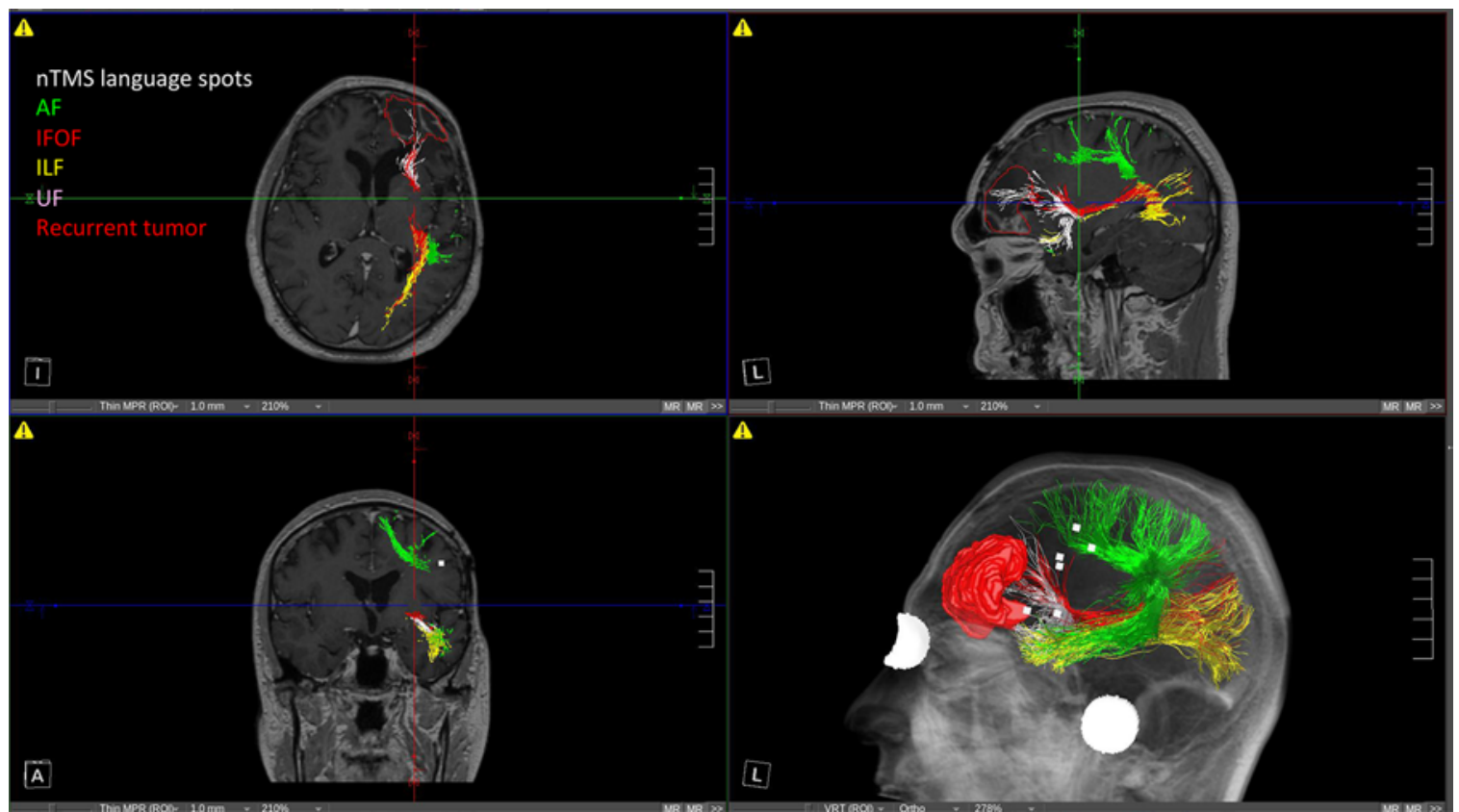

FIG. 7. Example of the intraoperative use of nTMS-based reconstruction of the language networks in the case of a recurrent left frontal high-grade glioma. The anterior portion of the IFOF (red) and UF (pink) seems to be infiltrated by the lesions, thereby increasing the risk of resection.

\section{Neurosurgical Use-Current Evidence}

The first paper describing the possibility of using nTMS for language mapping was published in $2012 .{ }^{37}$ Thereafter, preliminary studies were focused on the analysis of the accuracy of rTMS language mapping in comparison with the gold-standard DES, reporting a very high sensitivity and negative predictive value, but a low specificity and positive predictive value, especially for posterior language areas. ${ }^{44,74}$ The reliability of rTMS language mapping is lower than motor mapping, and can be influenced by different parameters, including the stimulation frequency and intensity, ${ }^{70}$ the task used, ${ }^{19}$ the coil positioning, ${ }^{70}$ and time between picture presentation and the beginning of the repetitive stimulation. ${ }^{33}$ Accordingly, a standardized protocol has been defined by experts to increase the accuracy of rTMS language mapping. ${ }^{29}$

Some authors also investigated the accuracy of rTMS versus fMRI for mapping language areas in comparison with the DES. In 2 consecutive studies Ille et al. reported that rTMS language mapping seems more sensitive but less specific in comparison with fMRI. ${ }^{25,26}$

The clinical usefulness of the combination of rTMS cortical language mapping with DTI fiber tracking has been demonstrated by several papers. ${ }^{41,52,56,72}$ Thanks to these studies, the nTMS-based reconstruction of the language network has also entered the clinical workflow prior to surgery of language-eloquent brain tumors ${ }^{71}$ (Video 2).

VIDEO 2. Example of nTMS-based language mapping and planning in a case of left temporal metastases from lung cancer. nTMS language spots (white spots) are overlapped to the most anterior and superior portion of the tumor (red). Conversely, subcortical white matter fascicles are dislocated medially and superiorly by the tumor, but they are not infiltrated (IFOF in purple, ILF in yellow, and UF in blue). The video shows clearly the presence of a safe surgical corridor between the tumor (located laterally) and subcortical fascicles (located medially) that may be used to achieve the maximal safe resection of the lesion, after intraoperative verification by DES. Copyright Giovanni Raffa. Published with permission. Click here to view.

Recently, some authors demonstrated the usefulness of rTMS language mapping especially in patients not eligible for awake surgery; $20,24,52,56$ a few other studies analyzed the impact of this approach in patients operated on using awake surgery. 22,27,62,69,71 Conclusions from all these studies were in accord that nTMS-based language mapping enables a reliable identification of the language network, and allows for a tailored surgical strategy that may result in a good functional and even oncological outcome. Nevertheless, literature evidence currently consists of observational studies only, and no randomized clinical trials have been either organized or published so far. In our center, nTMS language mapping was performed in 107 patients with language-eloquent tumors: gross-total resection was achieved in $72.9 \%$ of cases, and permanent language deficits were observed in $5.6 \%$ of patients (Table 1).

\section{Future Perspectives and Applications}

Some preliminary reports have demonstrated that nTMS motor and language mapping may be useful also for surgery of brain arteriovenous malformation $\mathrm{s}^{15,23,28}$ and of convexity meningiomas located in the central region. ${ }^{55} \mathrm{In}-$ terestingly, a similar approach has been recently reported as effective also before epilepsy surgery. ${ }^{36}$

More recently, some attempts to develop novel nTMSbased approaches for mapping other complex brain functions have been performed. New tasks have been implemented to enable the nTMS-based exploration of brain 
areas involved in more specific language domains such as reading, writing, verb generation, and action naming, ${ }^{19,61}$ as well as in other functions including tactile discrimination/ working memory, ${ }^{18}$ visuospatial, ${ }^{16,39}$ and calculation ${ }^{21,40}$ processes. The aim would be to reproduce by nTMS, in the preoperative period, the same settings we routinely have in the operating theater during awake surgery.

\section{Conclusions}

nTMS represents a novel electrophysiology-based imaging technique to perform and visualize, in a noninvasive way, preoperative brain mapping findings. Being based on neurophysiology, it provides valuable results that may anticipate findings otherwise available only by DES during surgery. Accordingly, nTMS might be considered a novel reliable tool for preoperative neurophysiological mapping of brain functional areas. This tool, combined with advanced imaging, might really impact surgical treatment of brain tumors located in eloquent areas.

\section{Acknowledgments}

This study has been funded by EU PON "R\&I" 2014-2020; Call AIM line 1 CUP 1839117-3. Recipient: Giovanni Raffa, MD, PhD, BIOMORF Department, University of Messina, Italy.

\section{References}

1. Catani M, Thiebaut de Schotten M: A diffusion tensor imaging tractography atlas for virtual in vivo dissections. Cortex 44:1105-1132, 2008

2. Chang EF, Raygor KP, Berger MS: Contemporary model of language organization: an overview for neurosurgeons. J Neurosurg 122:250-261, 2015

3. Coburger J, Musahl C, Henkes H, Horvath-Rizea D, Bittl $\mathrm{M}$, Weissbach C, et al: Comparison of navigated transcranial magnetic stimulation and functional magnetic resonance imaging for preoperative mapping in rolandic tumor surgery. Neurosurg Rev 36:65-76, 2013

4. Conti A, Raffa G, Granata F, Rizzo V, Germanò A, Tomasello F: Navigated transcranial magnetic stimulation for "somatotopic" tractography of the corticospinal tract. Neurosurgery 10 (Suppl 4):542-554, 2014

5. Corina DP, Loudermilk BC, Detwiler L, Martin RF, Brinkley JF, Ojemann G: Analysis of naming errors during cortical stimulation mapping: implications for models of language representation. Brain Lang 115:101-112, 2010

6. De Benedictis A, Duffau H: Brain hodotopy: from esoteric concept to practical surgical applications. Neurosurgery 68:1709-1723, 2011

7. Duffau H: The dangers of magnetic resonance imaging diffusion tensor tractography in brain surgery. World Neurosurg 81:56-58, 2014

8. Duffau H: The huge plastic potential of adult brain and the role of connectomics: new insights provided by serial mappings in glioma surgery. Cortex 58:325-337, 2014

9. Ekstrom A: How and when the fMRI BOLD signal relates to underlying neural activity: the danger in dissociation. Brain Res Brain Res Rev 62:233-244, 2010

10. Feindel W: The contributions of Wilder Penfield to the functional anatomy of the human brain. Hum Neurobiol 1:231234, 1982

11. Forster MT, Hattingen E, Senft C, Gasser T, Seifert V, Szelényi A: Navigated transcranial magnetic stimulation and functional magnetic resonance imaging: advanced adjuncts in preoperative planning for central region tumors. Neurosurgery 68:1317-1325, 2011
12. Forster MT, Senft C, Hattingen E, Lorei M, Seifert V, Szelényi A: Motor cortex evaluation by nTMS after surgery of central region tumors: a feasibility study. Acta Neurochir (Wien) 154:1351-1359, 2012

13. Frey D, Schilt S, Strack V, Zdunczyk A, Rösler J, Niraula B, et al: Navigated transcranial magnetic stimulation improves the treatment outcome in patients with brain tumors in motor eloquent locations. Neuro Oncol 16:1365-1372, 2014

14. Frey D, Strack V, Wiener E, Jussen D, Vajkoczy P, Picht T: A new approach for corticospinal tract reconstruction based on navigated transcranial stimulation and standardized fractional anisotropy values. Neuroimage 62:1600-1609, 2012

15. Germanò A, Raffa G, Conti A, Fiore P, Cardali SM, Esposito F, et al: Modern treatment of brain arteriovenous malformations using preoperative planning based on navigated transcranial magnetic stimulation: a revisitation of the concept of eloquence. World Neurosurg 131:371-384, 2019

16. Giglhuber K, Maurer S, Zimmer C, Meyer B, Krieg SM: Mapping visuospatial attention: the greyscales task in combination with repetitive navigated transcranial magnetic stimulation. BMC Neurosci 19:40, 2018

17. Groppa S, Oliviero A, Eisen A, Quartarone A, Cohen LG, Mall V, et al: A practical guide to diagnostic transcranial magnetic stimulation: report of an IFCN committee. Clin Neurophysiol 123:858-882, 2012

18. Hannula H, Neuvonen T, Savolainen P, Tukiainen T, Salonen $\mathrm{O}$, Carlson S, et al: Navigated transcranial magnetic stimulation of the primary somatosensory cortex impairs perceptual processing of tactile temporal discrimination. Neurosci Lett 437:144-147, 2008

19. Hauck T, Tanigawa N, Probst M, Wohlschlaeger A, Ille S, Sollmann N, et al: Task type affects location of languagepositive cortical regions by repetitive navigated transcranial magnetic stimulation mapping. PLoS One 10:e0125298, 2015

20. Hendrix P, Senger S, Simgen A, Griessenauer CJ, Oertel J: Preoperative rTMS language mapping in speech-eloquent brain lesions resected under general anesthesia: a pairmatched cohort study. World Neurosurg 100:425-433, 2017

21. Ille S, Drummer K, Giglhuber K, Conway N, Maurer S, Meyer B, et al: Mapping of arithmetic processing by navigated repetitive transcranial magnetic stimulation in patients with parietal brain tumors and correlation with postoperative outcome. World Neurosurg 114:e1016-e1030, 2018

22. Ille S, Kulchytska N, Sollmann N, Wittig R, Beurskens E, Butenschoen VM, et al: Hemispheric language dominance measured by repetitive navigated transcranial magnetic stimulation and postoperative course of language function in brain tumor patients. Neuropsychologia 91:50-60, 2016

23. Ille S, Picht T, Shiban E, Meyer B, Vajkoczy P, Krieg SM: The impact of nTMS mapping on treatment of brain AVMs. Acta Neurochir (Wien) 160:567-578, 2018

24. Ille S, Sollmann N, Butenschoen VM, Meyer B, Ringel F, Krieg SM: Resection of highly language-eloquent brain lesions based purely on rTMS language mapping without awake surgery. Acta Neurochir (Wien) 158:2265-2275, 2016

25. Ille S, Sollmann N, Hauck T, Maurer S, Tanigawa N, Obermueller T, et al: Combined noninvasive language mapping by navigated transcranial magnetic stimulation and functional MRI and its comparison with direct cortical stimulation. J Neurosurg 123:212-225, 2015

26. Ille S, Sollmann N, Hauck T, Maurer S, Tanigawa N, Obermueller T, et al: Impairment of preoperative language mapping by lesion location: a functional magnetic resonance imaging, navigated transcranial magnetic stimulation, and direct cortical stimulation study. J Neurosurg 123:314-324, 2015

27. Jung J, Lavrador JP, Patel S, Giamouriadis A, Lam J, Bhangoo R, et al: First United Kingdom experience of navigated 
transcranial magnetic stimulation in preoperative mapping of brain tumors. World Neurosurg 122:e1578-e1587, 2019

28. Kato N, Schilt S, Schneider H, Frey D, Kufeld M, Vajkoczy $\mathrm{P}$, et al: Functional brain mapping of patients with arteriovenous malformations using navigated transcranial magnetic stimulation: first experience in ten patients. Acta Neurochir (Wien) 156:885-895, 2014

29. Krieg SM, Lioumis P, Mäkelä JP, Wilenius J, Karhu J, Hannula $\mathrm{H}$, et al: Protocol for motor and language mapping by navigated TMS in patients and healthy volunteers; workshop report. Acta Neurochir (Wien) 159:1187-1195, 2017

30. Krieg SM, Picht T, Sollmann N, Bährend I, Ringel F, Nagarajan SS, et al: Resection of motor eloquent metastases aided by preoperative nTMS-based motor maps-comparison of two observational cohorts. Front Oncol 6:261, 2016

31. Krieg SM, Sabih J, Bulubasova L, Obermueller T, Negwer $\mathrm{C}$, Janssen I, et al: Preoperative motor mapping by navigated transcranial magnetic brain stimulation improves outcome for motor eloquent lesions. Neuro Oncol 16:1274-1282, 2014

32. Krieg SM, Shiban E, Buchmann N, Gempt J, Foerschler A, Meyer B, et al: Utility of presurgical navigated transcranial magnetic brain stimulation for the resection of tumors in eloquent motor areas. J Neurosurg 116:994-1001, 2012

33. Krieg SM, Tarapore PE, Picht T, Tanigawa N, Houde J, Sollmann N, et al: Optimal timing of pulse onset for language mapping with navigated repetitive transcranial magnetic stimulation. Neuroimage 100:219-236, 2014

34. Kuhnt D, Bauer MHA, Ganslandt O, Nimsky C: Functional imaging: where do we go from here? J Neurosurg Sci 57:111,2013

35. Larson E, Maddox RK, Lee AKC: Improving spatial localization in MEG inverse imaging by leveraging intersubject anatomical differences. Front Neurosci 8:330, 2014

36. Lehtinen H, Mäkelä JP, Mäkelä T, Lioumis P, Metsähonkala L, Hokkanen L, et al: Language mapping with navigated transcranial magnetic stimulation in pediatric and adult patients undergoing epilepsy surgery: comparison with extraoperative direct cortical stimulation. Epilepsia Open 3:224-235, 2018

37. Lioumis P, Zhdanov A, Mäkelä N, Lehtinen H, Wilenius J, Neuvonen T, et al: A novel approach for documenting naming errors induced by navigated transcranial magnetic stimulation. J Neurosci Methods 204:349-354, 2012

38. Mangraviti A, Casali C, Cordella R, Legnani FG, Mattei L, Prada F, et al: Practical assessment of preoperative functional mapping techniques: navigated transcranial magnetic stimulation and functional magnetic resonance imaging. Neurol Sci 34:1551-1557, 2013 (Erratum in Neurol Sci 35:501, 2014)

39. Maurer S, Giglhuber K, Sollmann N, Kelm A, Ille S, Hauck $\mathrm{T}$, et al: Non-invasive mapping of face processing by navigated transcranial magnetic stimulation. Front Hum Neurosci 11:4, 2017

40. Maurer S, Tanigawa N, Sollmann N, Hauck T, Ille S, BoeckhBehrens T, et al: Non-invasive mapping of calculation function by repetitive navigated transcranial magnetic stimulation. Brain Struct Funct 221:3927-3947, 2016

41. Negwer C, Sollmann N, Ille S, Hauck T, Maurer S, Kirschke JS, et al: Language pathway tracking: comparing nTMSbased DTI fiber tracking with a cubic ROIs-based protocol. J Neurosurg 126:1006-1014, 2017

42. Ottenhausen M, Krieg SM, Meyer B, Ringel F: Functional preoperative and intraoperative mapping and monitoring: increasing safety and efficacy in glioma surgery. Neurosurg Focus 38(1):E3, 2015

43. Pascual-Leone A, Bartres-Faz D, Keenan JP: Transcranial magnetic stimulation: studying the brain-behaviour relationship by induction of 'virtual lesions'. Philos Trans R Soc Lond B Biol Sci 354:1229-1238, 1999

44. Picht T, Krieg SM, Sollmann N, Rösler J, Niraula B, Neu- vonen T, et al: A comparison of language mapping by preoperative navigated transcranial magnetic stimulation and direct cortical stimulation during awake surgery. Neurosurgery 72:808-819, 2013

45. Picht T, Mularski S, Kuehn B, Vajkoczy P, Kombos T, Suess O: Navigated transcranial magnetic stimulation for preoperative functional diagnostics in brain tumor surgery. Neurosurgery 65 (6 Suppl):93-99, 2009

46. Picht T, Schmidt S, Brandt S, Frey D, Hannula H, Neuvonen $\mathrm{T}$, et al: Preoperative functional mapping for rolandic brain tumor surgery: comparison of navigated transcranial magnetic stimulation to direct cortical stimulation. Neurosurgery 69:581-588, 2011

47. Picht T, Schmidt S, Woitzik J, Suess O: Navigated brain stimulation for preoperative cortical mapping in paretic patients: case report of a hemiplegic patient. Neurosurgery 68:E1475-E1480, 2011

48. Picht T, Schulz J, Hanna M, Schmidt S, Suess O, Vajkoczy P: Assessment of the influence of navigated transcranial magnetic stimulation on surgical planning for tumors in or near the motor cortex. Neurosurgery 70:1248-1257, 2012

49. Pujol S, Wells W, Pierpaoli C, Brun C, Gee J, Cheng G, et al: The DTI challenge: toward standardized evaluation of diffusion tensor imaging tractography for neurosurgery. J Neuroimaging 25:875-882, 2015

50. Raffa G, Bährend I, Schneider H, Faust K, Germanò A, Vajkoczy P, et al: A novel technique for region and linguistic specific nTMS-based DTI fiber tracking of language pathways in brain tumor patients. Front Neurosci 10:552, 2016

51. Raffa G, Conti A, Scibilia A, Cardali SM, Esposito F, Angileri FF, et al: The impact of diffusion tensor imaging fiber tracking of the corticospinal tract based on navigated transcranial magnetic stimulation on surgery of motor-eloquent brain lesions. Neurosurgery 83:768-782, 2018

52. Raffa G, Conti A, Scibilia A, Sindorio C, Quattropani MC, Visocchi M, et al: Functional reconstruction of motor and language pathways based on navigated transcranial magnetic stimulation and DTI fiber tracking for the preoperative planning of low grade glioma surgery: a new tool for preservation and restoration of eloquent networks. Acta Neurochir Suppl 124:251-261, 2017

53. Raffa G, Germanò A, Tomasello F: Letter to the editor regarding "First United Kingdom Experience of Navigated Transcranial Magnetic Stimulation in Preoperative Mapping of Brain Tumors". World Neurosurg 125:549-550, 2019

54. Raffa G, Picht T, Angileri FF, Youssef M, Conti A, Esposito $\mathrm{F}$, et al: Surgery of malignant motor-eloquent gliomas guided by sodium-fluorescein and navigated transcranial magnetic stimulation: a novel technique to increase the maximal safe resection. J Neurosurg Sci [epub ahead of print], 2019

55. Raffa G, Picht T, Scibilia A, Rösler J, Rein J, Conti A, et al: Surgical treatment of meningiomas located in the rolandic area: the role of navigated transcranial magnetic stimulation for preoperative planning, surgical strategy, and prediction of arachnoidal cleavage and motor outcome. J Neurosurg [epub ahead of print June 14, 2019. DOI: 10.3171/2019.3.JNS183411]

56. Raffa G, Quattropani MC, Scibilia A, Conti A, Angileri FF, Esposito F, et al: Surgery of language-eloquent tumors in patients not eligible for awake surgery: the impact of a protocol based on navigated transcranial magnetic stimulation on presurgical planning and language outcome, with evidence of tumor-induced intra-hemispheric plasticity. Clin Neurol Neurosurg 168:127-139, 2018

57. Raffa G, Scibilia A, Conti A, Cardali SM, Rizzo V, Terranova $\mathrm{C}$, et al: Multimodal surgical treatment of high-grade gliomas in the motor area: the impact of the combination of navigated transcranial magnetic stimulation and fluoresceinguided resection. World Neurosurg 128:e378-e390, 2019 
58. Raffa G, Scibilia A, Conti A, Ricciardo G, Rizzo V, Morelli A, et al: The role of navigated transcranial magnetic stimulation for surgery of motor-eloquent brain tumors: a systematic review and meta-analysis. Clin Neurol Neurosurg 180:7-17, 2019

59. Raffa G, Scibilia A, Germanò A, Conti A: nTMS-based DTI fiber tracking of motor pathways, in Krieg SM (ed): Navigated Transcranial Magnetic Stimulation in Neurosurgery. Cham: Springer International Publishing, 2017, pp 97-114

60. Rizzo V, Terranova C, Conti A, Germanò A, Alafaci C, Raffa $\mathrm{G}$, et al: Preoperative functional mapping for rolandic brain tumor surgery. Neurosci Lett 583:136-141, 2014

61. Rogić Vidaković M, Gabelica D, Vujović I, Šoda J, Batarelo N, Džimbeg A, et al: A novel approach for monitoring writing interferences during navigated transcranial magnetic stimulation mappings of writing related cortical areas. J Neurosci Methods 255:139-150, 2015

62. Rosenstock T, Picht T, Schneider H, Koch A, Thomale UW: Left perisylvian tumor surgery aided by TMS language mapping in a 6-year-old boy: case report. Childs Nerv Syst 35:175-181, 2019

63. Rossini PM, Barker AT, Berardelli A, Caramia MD, Caruso G, Cracco RQ, et al: Non-invasive electrical and magnetic stimulation of the brain, spinal cord and roots: basic principles and procedures for routine clinical application. Report of an IFCN committee. Electroencephalogr Clin Neurophysiol 91:79-92, 1994

64. Rossini PM, Burke D, Chen R, Cohen LG, Daskalakis Z, Di Iorio R, et al: Non-invasive electrical and magnetic stimulation of the brain, spinal cord, roots and peripheral nerves: basic principles and procedures for routine clinical and research application. An updated report from an I.F.C.N. Committee. Clin Neurophysiol 126:1071-1107, 2015

65. Sanai N, Berger MS: Intraoperative stimulation techniques for functional pathway preservation and glioma resection. Neurosurg Focus 28(2):E1, 2010

66. Scibilia A, Conti A, Raffa G, Granata F, Abbritti RV, Priola SM, et al: Resting-state fMR evidence of network reorganization induced by navigated transcranial magnetic repetitive stimulation in phantom limb pain. Neurol Red 40:241-248, 2018

67. Scibilia A, Raffa G, Rizzo V, Quartarone A, Visocchi M, Germanò A, et al: Intraoperative neurophysiological monitoring in spine surgery: a significant tool for neuronal protection and functional restoration. Acta Neurochir Suppl 124:263270, 2017

68. Scibilia A, Terranova C, Rizzo V, Raffa G, Morelli A, Esposito $\mathrm{F}$, et al: Intraoperative neurophysiological mapping and monitoring in spinal tumor surgery: sirens or indispensable tools? Neurosurg Focus 41(2):E18, 2016

69. Sollmann N, Ille S, Hauck T, Maurer S, Negwer C, Zimmer $\mathrm{C}$, et al: The impact of preoperative language mapping by repetitive navigated transcranial magnetic stimulation on the clinical course of brain tumor patients. BMC Cancer 15:261, 2015
70. Sollmann N, Ille S, Obermueller T, Negwer C, Ringel F, Meyer B, et al: The impact of repetitive navigated transcranial magnetic stimulation coil positioning and stimulation parameters on human language function. Eur J Med Res 20:47, 2015

71. Sollmann N, Kelm A, Ille S, Schröder A, Zimmer C, Ringel F, et al: Setup presentation and clinical outcome analysis of treating highly language-eloquent gliomas via preoperative navigated transcranial magnetic stimulation and tractography. Neurosurg Focus 44(6):E2, 2018

72. Sollmann N, Negwer C, Ille S, Maurer S, Hauck T, Kirschke JS, et al: Feasibility of nTMS-based DTI fiber tracking of language pathways in neurosurgical patients using a fractional anisotropy threshold. J Neurosci Methods 267:45-54, 2016

73. Sollmann N, Picht T, Mäkelä JP, Meyer B, Ringel F, Krieg SM: Navigated transcranial magnetic stimulation for preoperative language mapping in a patient with a left frontoopercular glioblastoma. J Neurosurg 118:175-179, 2013

74. Tarapore PE, Findlay AM, Honma SM, Mizuiri D, Houde JF, Berger MS, et al: Language mapping with navigated repetitive TMS: proof of technique and validation. Neuroimage 82:260-272, 2013

75. Tomasello F, Angileri FF, Conti A, Scibilia A, Cardali S, La Torre D, et al: Petrosal meningiomas: factors affecting outcome and the role of intraoperative multimodal assistance to microsurgery. Neurosurgery 84:1313-1324, 2019

76. Villanueva-Meyer JE, Mabray MC, Cha S: Current clinical brain tumor imaging. Neurosurgery 81:397-415, 2017

\section{Disclosures}

The authors report no conflict of interest concerning the materials or methods used in this study or the findings specified in this paper.

\section{Author Contributions}

Conception and design: Raffa, Germanò. Acquisition of data: Quattropani. Analysis and interpretation of data: Quattropani. Drafting the article: Raffa. Critically revising the article: all authors. Reviewed submitted version of manuscript: Quattropani. Approved the final version of the manuscript on behalf of all authors: Raffa. Study supervision: all authors.

\section{Supplemental Information \\ Videos}

Video 1. https://vimeo.com/363548245.

Video 2. https://vimeo.com/363548253.

\section{Correspondence}

Giovanni Raffa: University of Messina, Italy.giovanni.raffa@ unime.it. 